\title{
IR-Induced Photoisomerization of Glycolic Acid Isolated in Low-Temperature Inert Matrices
}

\author{
Igor D. Reva,* Susana Jarmelo, Leszek Lapinski, and Rui Fausto \\ Departamento de Química, Universidade de Coimbra, 3004-535 Coimbra, Portugal, and \\ Institute of Physics, Polish Academy of Sciences, Al. Lotnikow 32/46, 02-668 Warsaw, Poland
}

Received: April 14, 2004; In Final Form: June 14, 2004

\begin{abstract}
Glycolic acid $\left(\mathrm{HO}-\mathrm{CH}_{2}-\mathrm{COOH}\right)$ monomers isolated in low-temperature argon and krypton matrices were studied using FTIR spectroscopy. The most stable SSC conformer of the compound dominated in the matrices immediately after their deposition. Upon broadband infrared irradiation, two minor conformers (GAC and AAT) were photogenerated. No other forms of glycolic acid were populated, either thermally or photochemically. Systematic monitoring of the progress of the IR-induced phototransformations (over a time scale of 20-140 min) allowed one to distinguish between the primary (GAC) and secondary (AAT) photoproducts. The assignment of experimental spectra to the different conformers was based on the results of IR irradiation of the samples and was assisted by the theoretical calculations carried out at the MP2/augcc-pVDZ level. Theoretical pathways for intramolecular rotation in the glycolic acid monomer were investigated at the MP2/6-31++G(d,p) level. Lack of conformers other than SSC, GAC, and AAT in the IR-irradiated matrices was explained in terms of energy barriers separating different forms of the compound.
\end{abstract}

\section{Introduction}

The conformers of glycolic acid $\left(\mathrm{HO}-\mathrm{CH}_{2}-\mathrm{COOH}\right.$; GA) can be defined by the values of the dihedral angles associated with three conformationally relevant torsional internal degrees of freedom: $\mathrm{HO}_{\mathrm{A}} \mathrm{CC}$ (syn, anti, gauche); $\mathrm{O}_{\mathrm{A}} \mathrm{CC}=\mathrm{O}$ (syn, anti); $\mathrm{O}=\mathrm{CO}_{\mathrm{C}} \mathrm{H}$ (cis, trans). To distinguish the oxygen atoms of the two $\mathrm{OH}$ groups, the subscripts $\mathrm{A}$ and $\mathrm{C}$ denote the oxygen atom belonging to the alcohol (A) or carboxylic (C) fragments, respectively. Seven conformers were theoretically predicted for the molecule of GA (Figure 1), with the SSC form corresponding to the lowest energy. This conformer is stabilized by an intramolecular hydrogen bond between the alcohol group and the carbonyl oxygen atom. The second and third most stable conformers (GAC and AAT, respectively) have relative energies of ca. 9 and $12 \mathrm{~kJ} \mathrm{~mol}^{-1}$. Theoretical geometry optimizations predict for the SSC and AAT conformers a planar heavy-atom framework, whereas the heavy-atom arrangement of the GAC form is predicted to be nonplanar. According to the theoretical calculations, the populations of the SSC, GAC, and AAT conformers in the gas phase, at room temperature, should be about $95 \%, 4 \%$, and $1 \%$, respectively, with other conformers being populated below the detection limits.

Recently, we were able to observe experimentally the SSC, AAT, and GAC conformers of GA. ${ }^{1}$ The vapor of the compound, with populations of the conformers corresponding to the gas-phase equilibrium, was trapped in low-temperature inert gas matrices, and bands due to the three conformers were observed in the FTIR spectra of the matrix-isolated compound. In that work, the GAC conformer, bearing an $\mathrm{O}_{\mathrm{A}} \mathrm{H} \cdots \mathrm{O}_{\mathrm{C}} \mathrm{H}$ intramolecular hydrogen bond, was unequivocally identified for the first time. The theoretically predicted energies of the remaining four conformers of GA are higher, and no experimental evidence of these forms has ever been reported.

* To whom correspondence should be addressed. E-mail: reva@qui.uc.pt.
In a previous matrix-isolation spectroscopy investigation, Hollenstein and collaborators ${ }^{2,3}$ studied the infrared spectra of several matrix-isolated isotopologues of GA. Besides the dominant IR features of the SSC conformer, present in the spectra of freshly prepared Ar matrices, these authors were able to observe, in the spectrum obtained after broadband IR irradiation of the matrix-isolated compound, a series of new emerging bands, which were assigned to the AAT conformer. However, the lack of observation in those experiments of the second most stable conformer (GAC) appeared intriguing to us. In fact, efficient IR-induced conversion of the most stable SSC conformer directly into the AAT form does not seem to be probable, taking into account that this process would require simultaneous change in all three internal rotation axes $\left(\mathrm{HO}_{\mathrm{A}^{-}}\right.$ $\mathrm{CC}, \mathrm{O}_{\mathrm{A}} \mathrm{CC}=\mathrm{O}$, and $\left.\mathrm{O}=\mathrm{CO}_{\mathrm{C}} \mathrm{H}\right)$. A rotamerization pathway involving an initial conversion from SSC to GAC and then from this form to AAT appears much more plausible, considering the relatively low predicted energy barriers for these two steps (ca. 20 and $40 \mathrm{~kJ} \mathrm{~mol}^{-1}$, respectively ${ }^{4}$ ). This would mean that, if the conversion of SSC into AAT occurs through the GAC intermediate, this latter form should also be detected, at least during the initial stages of the reaction (unless the intramolecular energy redistribution is much more efficient for the second step of the reaction than for the first one). That is why it seemed interesting to check if the bands appearing in the spectrum of the IR-irradiated matrix should be assigned only to the AAT form or to both GAC and AAT conformers.

In a series of recent works on IR-induced rotamerizations in carboxylic acids (such as formic, ${ }^{5}$ acetic, ${ }^{6}$ oxalic, ${ }^{7}$ malonic, ${ }^{8}$ and maleic ${ }^{9}$ acids), it has been shown that pumping of the $\mathrm{OH}$ stretching overtone vibrations is particularly efficient in promoting conformational changes. Such excitation requires energy of ca. $80-90 \mathrm{~kJ} \mathrm{~mol}^{-1}$, which is available under the unfiltered glowbar broadband IR irradiation conditions. The most stable form (SSC) and the sixth conformer of GA differ in energy by less than $20 \mathrm{~kJ} \mathrm{~mol}^{-1}$, and the seventh (highest energy) 
<smiles>CC(O)C(=O)O</smiles>

SSC $(0.0)$<smiles>CC(O)C(=O)O</smiles><smiles>C[C@@H](O)C(=O)O</smiles>

SST $\quad(19.5)$<smiles>CC(O)C(=O)O</smiles>

SAC* $^{*}(11.8)$

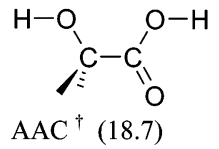<smiles>C[C@@H](O)C(=O)O</smiles>

$\operatorname{SAT}^{* *}(57.4)$

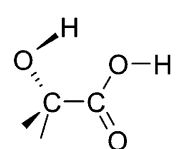

GAC $(9.2)$
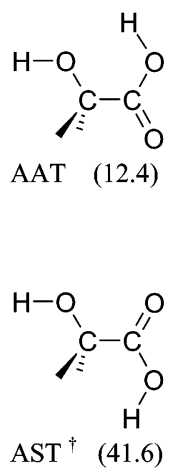

Figure 1. Schematic representation of conformers of glycolic acid. A, G, S, C, and T stand for anti, gauche, syn, cis, and trans. Three letters in the symbolic names of the conformers describe the orientation around the three dihedral angles, $\mathrm{H}-\mathrm{O}_{\mathrm{A}}-\mathrm{C}-\mathrm{C}, \mathrm{O}_{\mathrm{A}}-\mathrm{C}-\mathrm{C}=\mathrm{O}$, and $\mathrm{O}=\mathrm{C}-\mathrm{O}_{\mathrm{C}}-\mathrm{H}$, respectively. Alcohol and carboxyl groups are designated as $\mathrm{O}_{\mathrm{A}}$ and $\mathrm{O}_{\mathrm{C}}$, respectively. The $\mathrm{SSC}$ form is the global energy minimum. The SAC form with a planar heavy-atom backbone is the first-order saddle point (marked by asterisk) separating two nonplanar forms, GAC and G'AC. These minima are two symmetrically equivalent mirror forms. The SAT structure with a planar arrangement of heavy atoms is a second-order saddle point (marked by two asterisks). The remaining structures are true minima with planar arrangements of heavy atoms or very small deviations from planarity. Three structures (ASC, AAC, AST) designated by $\dagger$ occur as secondary energy minima defined by very low restraining barriers (see text). In the analysis, the latter conformations effectively act as transition states, and higher level MO computations might show that they are true transition states. Thus, symbol $\dagger$ shows that the corresponding structures are quasi-saddlepoint configurations. The relative energies (in $\mathrm{kJ} \mathrm{mol}^{-1}$, the zero-point vibrational energy not included) with respect to the SSC form, calculated at the MP2/aug-cc-pVDZ level, are shown in parentheses.

conformer has a relative energy of ca. $40 \mathrm{~kJ} \mathrm{~mol}^{-1}$ with respect to SSC, with all relative energies then being well below the $\mathrm{OH}$ stretching overtone excitation energy. Relevant energy barriers are also supposed to be lower than this energy. ${ }^{4}$ Hence, it could be expected that broadband IR excitation would be sufficient to populate all conformers of GA in the matrices. The lack of observation of all of the conformers of GA after IR irradiation of a matrix presents another interesting question.

Taking into consideration the above-mentioned open questions, in the present work, we reinvestigated the effects of broadband IR irradiation of matrix-isolated GA to search for experimental manifestations of conformers other than SSC and AAT. The experimental results are supported by extensive theoretical calculations undertaken at the MP2/6-31++G(d,p) level. Knowledge of the barrier heights for interconformational rearrangements was very important for the present study. In our previous work, we have shown that the relative energies obtained at the MP2/6-31++G(d,p) level compare well with those obtained at the MP2/aug-cc-pVDZ level. Thus, for consistency, all calculations reported in this work were performed at the MP2/ 6-31++G(d,p) level. In the microwave investigation of gaseous GA by Godfrey et al., ${ }^{4}$ only the barriers between selected conformers were studied. The set of considered conformers was restricted to those that could be populated thermally in the gaseous phase at $300{ }^{\circ} \mathrm{C}$. In the present study, barriers between all GA conformers were theoretically calculated.

\section{Experimental and Computational Methods}

Glycolic acid of spectroscopic purity grade was a commercial product obtained from Aldrich. Immediately before matrix deposition, the compound was additionally purified by pumping volatile impurities through the cryostat. A glass vacuum system and standard manometric procedures were used to deposit matrix gases (Air Liquide: argon N60, krypton N48), which were used without further purification. Gas deposition rate during sample preparation was ca. $10 \mathrm{mmol} \mathrm{h}^{-1}$. GA was codeposited from a specially designed Knudsen cell with shut-off possibility, the principal component of which is a SS-4 BMRG micrometer valve (NUPRO). Further details of the experimental setup can be found elsewhere. ${ }^{10}$

The IR spectra in the range $4000-400 \mathrm{~cm}^{-1}$ were obtained using a Mattson (Infinity 60AR series) FTIR spectrometer equipped with a deuterated triglycine sulfate (DTGS) detector and $\mathrm{Ge} / \mathrm{KBr}$ beam splitter. Data collection was performed with $0.5 \mathrm{~cm}^{-1}$ spectral resolution. All experiments were done based on an APD Cryogenics closed-cycle helium refrigeration system with a DE-202A expander.

The samples were irradiated (through the outer $\mathrm{KBr}$ window of the cryostat) with infrared light emitted by a kanthal wire electrically heated to an orange glow.

The theoretical calculations were performed with the Gaussian 98 program package ${ }^{11}$ at the MP2 level of theory with the $6-31++G(d, p)$ and aug-cc-pVDZ basis sets. ${ }^{12,13}$ The geometry optimizations were performed using the TIGHT optimization criteria and followed by frequency calculations to check the nature of the calculated structures. The frequencies calculated at the MP2/aug-cc-pVDZ level were used to assist the analysis of the experimental spectra and were scaled using two factors: 0.993 below $2000 \mathrm{~cm}^{-1}$ and 0.955 above $2000 \mathrm{~cm}^{-1}$ (i.e., for $\mathrm{OH}$ stretching). These scaling factors give the best agreement between the predicted and observed frequencies, especially in the $\mathrm{OH}$ and $\mathrm{C}=\mathrm{O}$ stretching regions. An important part of this study represents theoretical calculations of the reaction pathways for conformational interconversions. The energy barriers separating different conformers of GA were calculated in this work at the $6-31++G(d, p)$ level of theory unless specified otherwise.

\section{Results and Discussion}

Theoretical Calculations: Relative Energies of the Conformers and Barriers to Internal Rotation. The theoretically calculated relative energies (including ZPVE contributions) of the conformers of GA as well as the heights of the barriers for conversion of one conformer into the other are presented in Table 1.

Figure 2 shows the relaxed potential energy scan for rotation around the $\mathrm{C}-\mathrm{C}$ bond in $\mathrm{GA}$, that is, for conversion between the two most stable forms (SSC and GAC). Note that the MP2/ $6-31++\mathrm{G}(\mathrm{d}, \mathrm{p})$ calculations predict the SSC planar structure as being a first-order saddle point between two equivalent-bysymmetry nonplanar forms with the $\mathrm{O}=\mathrm{C}-\mathrm{C}-\mathrm{O}_{\mathrm{A}}$ dihedral angle equal to $\pm 12.5^{\circ}$. However, calculations performed with the larger aug-cc-pVDZ basis set ${ }^{1}$ predict the planar framework structure as the true minimum, which indicates that the small barrier found with the $6-31++G(d, p)$ basis set at the planar geometry might result from truncation of the basis set and might be numerically insignificant. The energy of the planar form, calculated at the MP2/6-31++G(d,p) level, is only $0.2 \mathrm{~kJ} \mathrm{~mol}^{-1}$ above that of the true minima (i.e., well below the zero-point energy for the $\mathrm{C}-\mathrm{C}$ torsional coordinates of these structures $\left(41.8 \mathrm{~cm}^{-1}\right.$ or $\left.\left.0.5 \mathrm{~kJ} \mathrm{~mol}^{-1}\right)\right)$. Hence, in any case, only the planar form of SSC is of practical interest. Comparison of our data 
TABLE 1: Energies Relative to the SSC Form (ZPVE Included, in $\mathrm{kJ} \mathrm{mol}^{-1}$ ) Calculated at the MP2/aug-cc-pVDZ and MP2/ 6-31 $++G(d, p)$ Levels of Theory for Different Glycolic Acid Conformers, and Barriers (in $\mathbf{k J ~ m o l}^{-1}$ ) Separating These Conformers by a Single Intramolecular Rotation Calculated at the MP2/6-31++G(d,p) Level ${ }^{a}$

\begin{tabular}{|c|c|c|c|c|c|c|c|c|c|c|}
\hline \multirow{2}{*}{$\begin{array}{l}\text { initial } \\
\text { conformer }\end{array}$} & \multicolumn{2}{|c|}{$\begin{array}{l}\text { MP2 relative } \\
\text { energies }\end{array}$} & \multicolumn{8}{|c|}{ target conformer } \\
\hline & $b$ & $c$ & $\overline{\mathrm{SSC}}$ & GAC & $\mathrm{G}^{\prime} \mathrm{AC}$ & AAT & ASC & AAC & SST & AST \\
\hline SSC & 0.0 & 0.0 & & 17.8 & 17.8 & & 19.0 & & 53.0 & \\
\hline GAC & 9.5 & 7.9 & 11.0 & & 4.4 & 49.7 & & 13.2 & & \\
\hline $\mathrm{G}^{\prime} \mathrm{AC}$ & 9.5 & 7.9 & 14.7 & 4.4 & & 58.9 & & 13.2 & & \\
\hline AAT & 12.0 & 13.3 & & & & & & 53.0 & 35.5 & \\
\hline ASC & 16.6 & 17.3 & 0.8 & & & & & & & \\
\hline AAC & 18.6 & 19.6 & & 1.0 & 1.0 & 45.9 & & & & \\
\hline SST & 19.1 & 24.7 & 27.7 & & & 12.7 & & & & 23.7 \\
\hline AST & 40.1 & 47.1 & & & & & & & 0.1 & \\
\hline
\end{tabular}

${ }^{a}$ For the GAC conformer, the barriers depend on direction of rotation. To distinguish between the two different possible directions, this form is shown in the table along with its mirror image G'AC. The starting conformers are presented in the first column, and the resulting conformers are in the column head row. If one of the conformers was found to have a low barrier $\left(1.0 \mathrm{~kJ} \mathrm{~mol}^{-1}\right.$ or less) separating it from another energetically more stable form, further barriers were not calculated. ${ }^{b}$ Calculation at the MP2/aug-cc-pVDZ level; energy of the SSC form is equal to -303.517652 Hartrees. ${ }^{c}$ Calculation at the MP2/6-31++G(d,p) level; energy of the SSC form is equal to -303.435695 Hartrees.
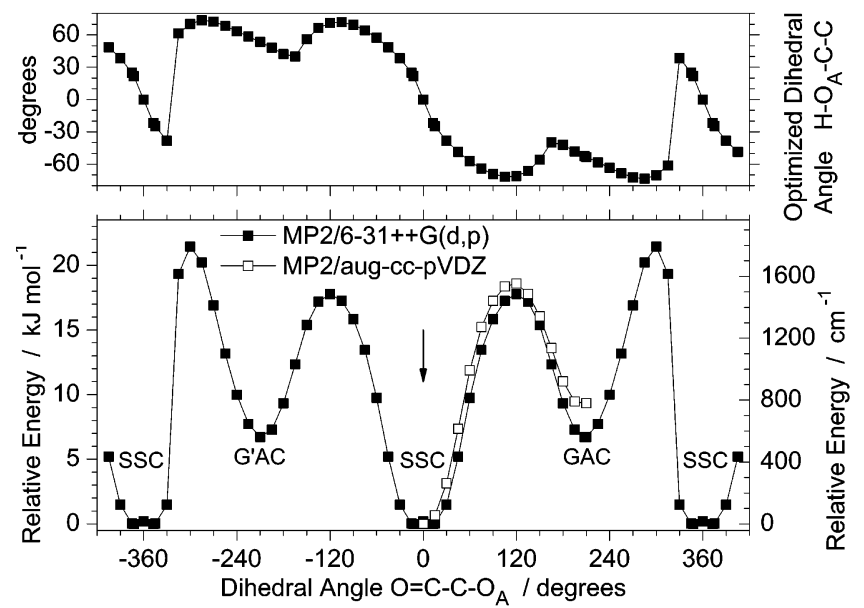

Figure 2. MP2/6-31++G(d,p) (black squares) and MP2/aug-cc-pVDZ (open squares) relaxed potential energy scans for rotation around the $\mathrm{C}-\mathrm{C}$ bond in glycolic acid. The scans were performed in both directions starting from the geometry designated by the arrow. The fragment between the GAC and SSC forms additionally was scanned in the opposite direction (from GAC to SSC). This scan resulted in the same barrier height (i.e., varying the reaction coordinates between $0^{\circ}$ and $240^{\circ}$ produces reversible changes in geometry and energy). Irregularities around $330^{\circ}$ and $-330^{\circ}$ are irreversible. Note that the scan in different directions, starting at the SSC form, produces either the GAC form or its mirror image $\mathrm{G}^{\prime} \mathrm{AC}$. This fact is obvious from the upper frame, which shows values of the optimized $\mathrm{HO}_{\mathrm{A}} \mathrm{CC}$ dihedral angle in each point. For convenience, energy units in the lower frame are represented in this and subsequent figures both in kilojoules per mole (left) and in reciprocal centimeters (right).

(Table 1) with data reported in the literature ${ }^{4}$ shows that the increase of the basis set leads to a slight decrease of the energy barriers (at the MP2/6-31G(d,p) level, the lowest energy barriers for conversion from SSC to GAC and from GAC to SSC were predicted to be 19.8 and $11.6 \mathrm{~kJ} \mathrm{~mol}^{-1}$, respectively). The most relevant result that can be extracted from the analysis of the potential energy profile shown in Figure 2 is the fact that conformer GAC is predicted to be easily accessible from the lowest energy SSC conformer upon IR excitation. According to the theoretical predictions, the process in the opposite direction should also occur easily.

Figure 3 shows the relaxed potential energy scan for rotation around the $\mathrm{C}-\mathrm{O}_{\mathrm{C}}$ bond, starting from the second lowest energy form (GAC). Because the initial geometry is asymmetric, the rotation around the $\mathrm{C}-\mathrm{O}_{\mathrm{C}}$ bond was investigated in both directions. The resulting energy barriers were found to be
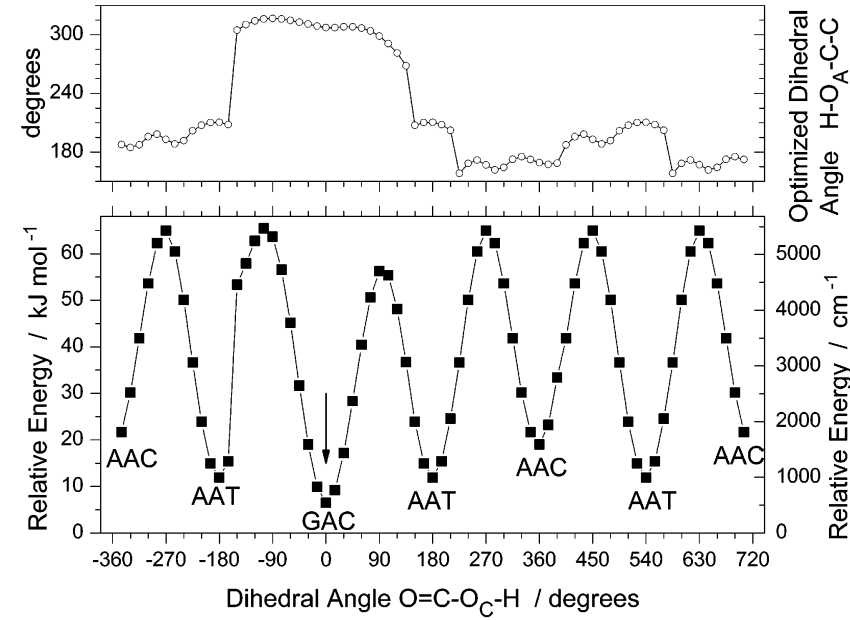

Figure 3. MP2/6- $31++G(d, p)$ relaxed potential energy scan for rotation around the $\mathrm{C}-\mathrm{O}_{\mathrm{C}}$ bond in glycolic acid, starting from the second lowest energy form (GAC) and optimized value of the $\mathrm{HO}_{\mathrm{A}} \mathrm{CC}$ dihedral angle at each point (upper frame). The vertical arrow indicates the initial geometry for this scan. The zero-level relative energy corresponds to the energy of the global minimum (SSC form). Because the initial geometry is asymmetric, the rotation around the $\mathrm{C}-\mathrm{O}_{\mathrm{C}}$ bond was investigated in both directions.

dependent on the direction of the rotation. However, both clockwise and counterclockwise scans result in conversion of GAC into the AAT form after completing a half turn. Indeed, when the $\mathrm{O}=\mathrm{C}-\mathrm{O}_{\mathrm{C}}-\mathrm{H}$ dihedral angle approaches either $180^{\circ}$ or $-180^{\circ}$, the alcohol group quickly switches its configuration to the anti position with respect to the $\mathrm{C}-\mathrm{C}$ bond (the optimized value of the $\mathrm{HO}_{\mathrm{A}} \mathrm{CC}$ dihedral angle stays in the vicinity of $180^{\circ}$; see the upper frame in Figure 3), leading to the AAT conformer. A full turn does not re-establish the starting GAC geometry, but results in the $\mathrm{AAC}$ conformation (the $\mathrm{HO}_{\mathrm{A}} \mathrm{CC}$ dihedral angle keeps a value around $180^{\circ}$ ). Continuation of the scan in the same direction (a second full turn) results again in the AAT and $\mathrm{AAC}$ forms, and it might seem that the process is irreversible with respect to the initial GAC geometry. However, the AAT form can convert back to the GAC form via the AAC intermediate. The AAC conformer is connected to the GAC form (by internal rotation of the alcohol group) by the energy barrier of $1.0 \mathrm{~kJ} \mathrm{~mol}^{-1}$ (Figure 4). Such a low barrier is not sufficient to trap the AAC form in matrices, and even if it were photoproduced upon irradiation of the matrix, its observation would be improbable under our experimental conditions. The calculated barriers separating the AAT form from AAC and 


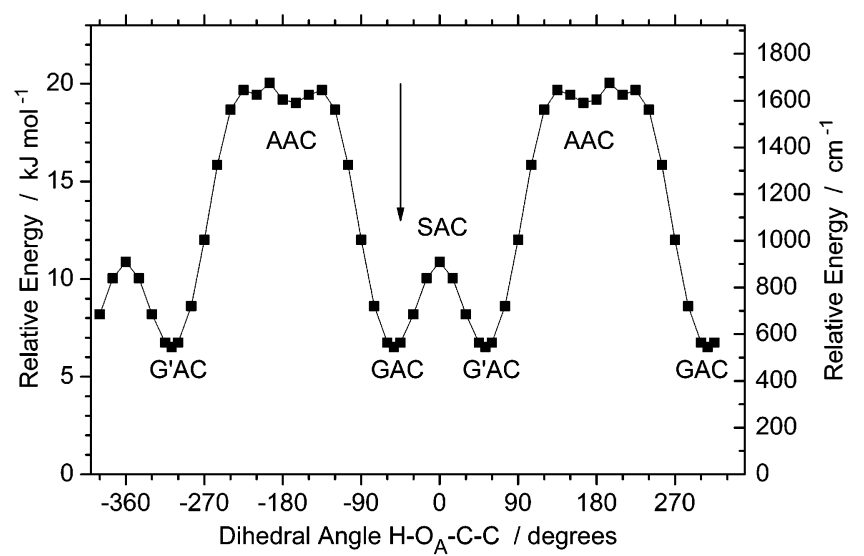

Figure 4. MP2/6-31++G(d,p) relaxed potential energy scan for rotation around the $\mathrm{O}_{\mathrm{A}}-\mathrm{C}$ bond in GA, starting from the second lowest energy form (GAC). The vertical arrow indicates the initial geometry for this scan. The zero-level relative energy corresponds to the energy of the global minimum (SSC form). Because the initial geometry is asymmetric, the rotation around the $\mathrm{O}_{\mathrm{A}}-\mathrm{C}$ bond was investigated in both directions. Note also that, upon completion of the full turn, the molecule returns to the initial geometry and passes also through the geometry that is its mirror equivalent ( $\left.\mathrm{G}^{\prime} \mathrm{AC}\right)$. The SAC conformation corresponds to the transition state for the interconversion between the two equivalent-by-symmetry GAC conformers and has an energy higher than these forms by $4.4 \mathrm{~kJ} \mathrm{~mol}^{-1}\left(364 \mathrm{~cm}^{-1}\right)$.

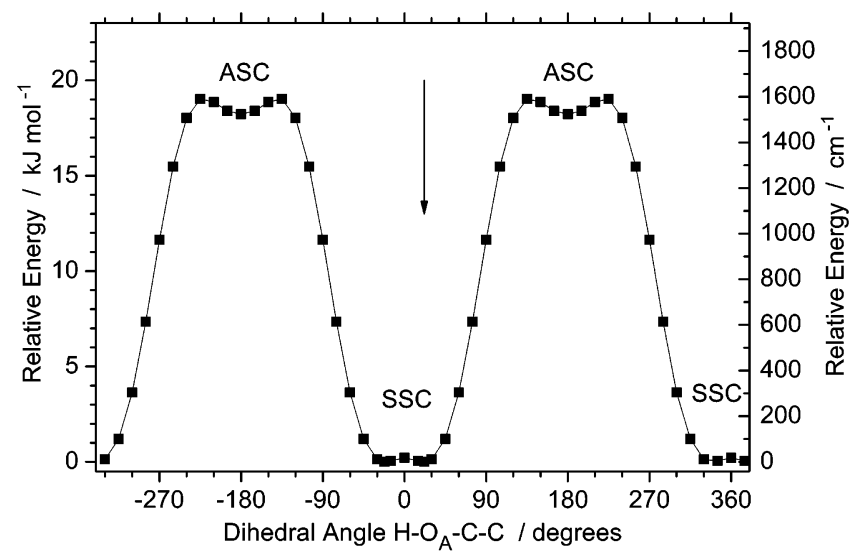

Figure 5. MP2/6-31++G(d,p) relaxed potential energy scan for rotation around the $\mathrm{O}_{\mathrm{A}}-\mathrm{C}$ bond in $\mathrm{GA}$, starting from the global energy minimum (SSC). The vertical arrow indicates the initial geometry for this scan. The rotation around the $\mathrm{O}_{\mathrm{A}}-\mathrm{C}$ bond was investigated in both directions and produced equal results.

GAC are relatively high, above $45 \mathrm{~kJ} \mathrm{~mol}^{-1}$ (Table 1). On the whole, the results shown in Figure 3 demonstrate that conversion from GAC to AAT is still possible under IR excitation.

From the discussion of the data presented in Figures 2-4, it can be concluded that IR pumping of the most stable SSC conformer should lead to photoproduction of GAC and AAT, and that the AAC form should not be detectable. Let us now examine the possibility of observing the remaining conformers (ASC, SST, and AST) in the IR-irradiated matrices. These conformers are the three highest energy forms of GA (see Table 1) but, in principle, are still accessible upon broadband IR irradiation, as referred to in the Introduction.

Figure 5 shows the relaxed potential energy scan for rotation around the $\mathrm{O}_{\mathrm{A}}-\mathrm{C}$ bond, starting from the global energy minimum (SSC). The ASC form is separated from the SSC conformer by a barrier of only $0.8 \mathrm{~kJ} \mathrm{~mol}^{-1}\left(66 \mathrm{~cm}^{-1}\right)$. With such a low barrier, the ASC conformer is unlikely to be trapped in matrices. Even if it could be photochemically generated, it would decay back to the most stable conformer.

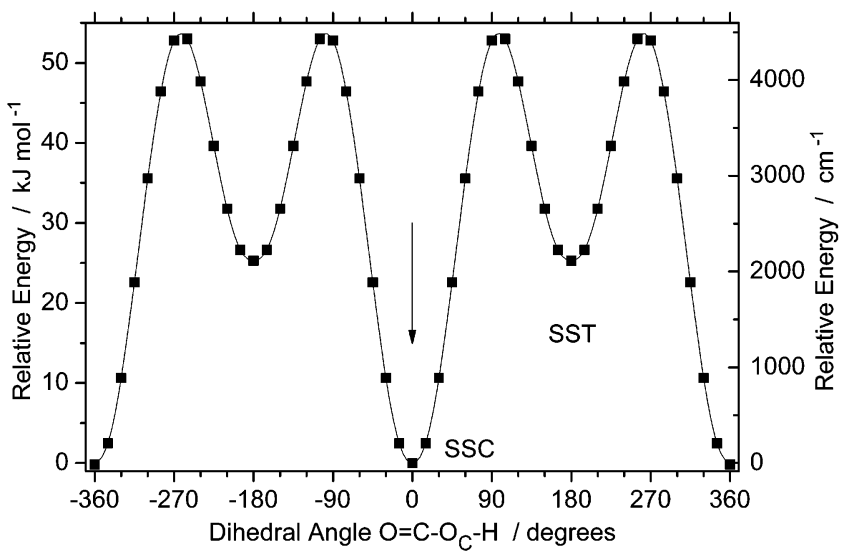

Figure 6. MP2/6-31++G(d,p) relaxed potential energy scan around the $\mathrm{C}-\mathrm{O}_{\mathrm{C}}$ bond, starting from the global minimum (SSC). The vertical arrow indicates the initial geometry for this scan. Barriers for rotation in the clockwise or counterclockwise direction are equal.

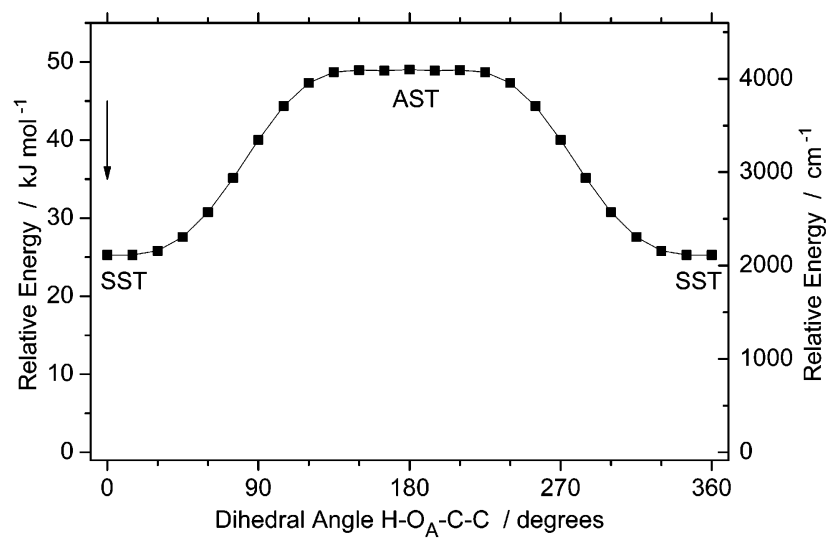

Figure 7. MP2/6-31+ $+\mathrm{G}(\mathrm{d}, \mathrm{p})$ relaxed potential energy scan for rotation around the $\mathrm{O}_{\mathrm{A}}-\mathrm{C}$ bond in GA, starting from the SST form. The zero-level relative energy corresponds to the energy of the global minimum (SSC form). The vertical arrow indicates the initial geometry for this scan.

The situation is less obvious as to what concerns the SST conformer. Internal rotation around the $\mathrm{C}-\mathrm{O}_{\mathrm{C}}$ bond in carboxylic acids usually produces forms that are separated by relatively high barriers. ${ }^{5,6}$ For GA, this has already become clear in Figure 3 , where the potential energy scan around this bond, starting from the GAC conformer, was shown. If the starting conformation is the SSC form (the global minimum), the potential energy profile corresponding to rotation around the $\mathrm{C}-\mathrm{O}_{\mathrm{C}}$ bond connects the SSC and SST conformers (Figure 6). The SST to SSC energy barrier is $27.7 \mathrm{~kJ} \mathrm{~mol}^{-1}$ (or $2315 \mathrm{~cm}^{-1}$ ). The calculated MP4/cc-pVTZ barrier between the similar cis and trans conformers of acetic acid is higher $\left(2549.4 \mathrm{~cm}^{-1}\right),{ }^{14}$ and the higher-energy cis acetic acid decays back to the trans form in a minute time scale (as measured in the dark in solid argon at $8 \mathrm{~K}){ }^{6}$ By analogy, the SST form of glycolic acid may be expected to decay to SSC in low-temperature matrices. Also, the possibility of trapping the SST form in matrices may depend on the height of the barriers separating this form from species other than SSC. These barriers are shown in Figures 7 and 8, where the relevant potential energy profiles for internal rotation around the $\mathrm{O}_{\mathrm{A}}-\mathrm{C}$ and $\mathrm{C}-\mathrm{C}$ bonds are presented.

Internal rotation around the $\mathrm{O}_{\mathrm{A}}-\mathrm{C}$ bond, starting from SST (see Figure 7), connects this conformer to the highest energy conformer (AST). The calculated energy barrier for the reverse reaction is only $7 \mathrm{~cm}^{-1}$ (less than $0.1 \mathrm{~kJ} \mathrm{~mol}^{-1}$ ). This result reveals clearly that the possibility of trapping the AST form in 


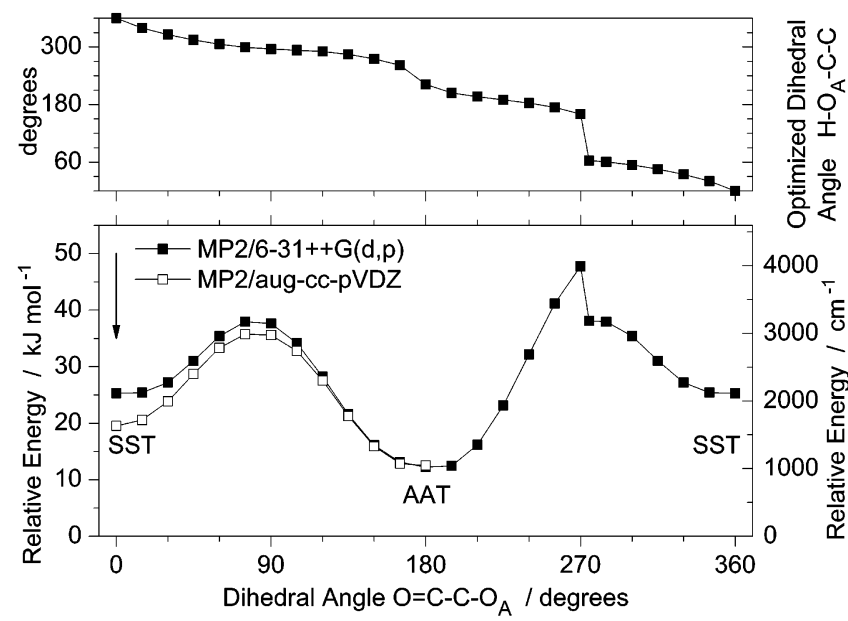

Figure 8. MP2/6-31++G(d,p) (black squares) and MP2/aug-cc-pVDZ (open squares) relaxed potential energy scans for rotation around the $\mathrm{C}-\mathrm{C}$ bond in GA, starting from the SST form (at the geometry designated by arrow). The zero-level relative energy corresponds to the energy of the global minimum (SSC form). The irregularity around $270^{\circ}$ is commented on in the text.

the matrices can be safely excluded, as it readily converts back to the SST form. On the other hand, the question of the possibility of observation of the SST conformer remains open.

In Figure 8, the potential energy profile for rotation around the $\mathrm{C}-\mathrm{C}$ bond, starting from SST, is depicted. It is also noteworthy that, while the $\mathrm{O}=\mathrm{C}-\mathrm{C}-\mathrm{O}_{\mathrm{A}}$ angle makes a full turn, the alcohol group also completes a full turn, from syn to anti and again to the syn position (upper frame in Figure 8). In this case, a synchronous rotation around both the $\mathrm{C}-\mathrm{C}$ and $\mathrm{O}_{\mathrm{A}}-$ $\mathrm{C}$ bonds shall occur, interconverting the SST and AAT conformers to each other. The barrier separating the SST form from the lower-energy AAT conformer is $12.7 \mathrm{~kJ} \mathrm{~mol}^{-1}$ (1058 $\left.\mathrm{cm}^{-1}\right){ }^{15}$ This barrier is even lower than that associated with the SST to SSC conversion (27.7 $\mathrm{kJ} \mathrm{mol}^{-1}$; see Figure 6), indicating that the SST form can relax more easily to AAT than to SSC. The possibility of observing a conformer in an experiment depends on the height of the energy barrier separating it from another lower-energy form. In our previous study on cyanoacetic acid, we have experimentally found that a barrier of $3 \mathrm{~kJ} \mathrm{~mol}^{-1}$ was not sufficient to trap a higher-energy conformer in low-temperature matrices. On the other hand, in the microwave studies of glycolic acid, ${ }^{4}$ it was deduced that a barrier of ca. $1000 \mathrm{~cm}^{-1}\left(12 \mathrm{~kJ} \mathrm{~mol}^{-1}\right)$ would not be enough to prevent conformational relaxation at $300{ }^{\circ} \mathrm{C}$ in the gaseous phase. However, it should be noted that in matrix-isolation experiments the energy barriers, which are required for observation of a conformer, are usually lower ${ }^{16}$ than in microwave studies. ${ }^{17,18}$ In the case of glycolic acid, its GAC conformer is separated from the ground-state SSC form by a barrier of $11 \mathrm{~kJ}$ mol $^{-1}$ (Figure 2) and was successfully observed in matrices, ${ }^{1}$ while it was not observed in the gas phase. ${ }^{4}$ Hence, the definite answer about the possibility of trapping the SST form should be sought in the experiment.

Experimental Results: Matrix Isolation Spectra. The survey experimental FTIR spectrum of glycolic acid trapped in an argon matrix, collected immediately after deposition of the sample, is presented in Figure 1S in the Supporting Information. The experimental spectrum corresponds to a sample prepared in such a manner that the matrix contained the smallest possible populations of the higher-energy forms. For this purpose, the compound was sublimated at the lowest possible nozzle temperature (room temperature, ca. 297 K). The experimental

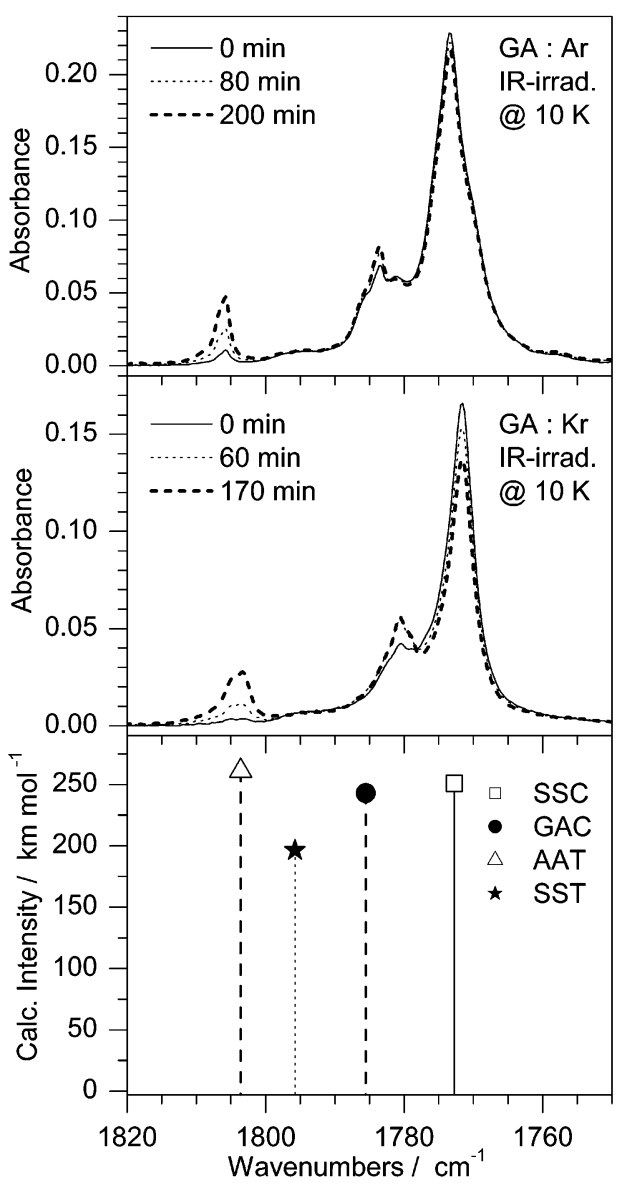

Figure 9. Spectra of GA isolated in argon (upper frame) and krypton (middle frame) matrices and the MP2/aug-cc-pVDZ-predicted (lower frame) spectra for SSC, GAC, AAT, and SST forms (carbonyl stretching region). Calculated frequencies are scaled by 0.993 .

spectrum of the freshly deposited, nonirradiated sample shows overall good agreement with the theoretically calculated spectrum of the SSC form (Figure 1S in Supporting Information). The assignments of the observed bands are presented in Table 1S (Supporting Information). Internal coordinates used in normal coordinate analyses are listed in Table 2S (Supporting Information).

From the theoretical results discussed in the previous section, it could be concluded that, following broadband IR pumping of the most stable SSC conformer of GA, we can confidently expect to observe production of the AAT and GAC conformers. In addition, the possibility of observing experimentally the SST conformer also could not be excluded on the basis of theoretical grounds.

The $1820-1750 \mathrm{~cm}^{-1}$ region (carbonyl stretching region) of the IR spectra of GA isolated in argon and krypton matrices is compared in Figure 9 with the spectra theoretically predicted (at the MP2/aug-cc-pVDZ level) for the SSC, GAC, AAT, and SST conformers of the compound. At room temperature, the relative population of SST should amount to $0.04 \%$ in the equilibrium gaseous mixture of conformers. ${ }^{1}$ Such a small population cannot be traced experimentally under the conditions of the present study. However, if during irradiation this conformer is produced, the bands due to this form should be easily distinguishable. The samples were deposited (solid line) and subsequently irradiated (dotted and dashed lines) at the lowest possible temperature of the optical substrate $(9-10 \mathrm{~K})$ to maximize the probability of trapping in the matrices forms with small relaxation barriers to the neighboring lower-energy 


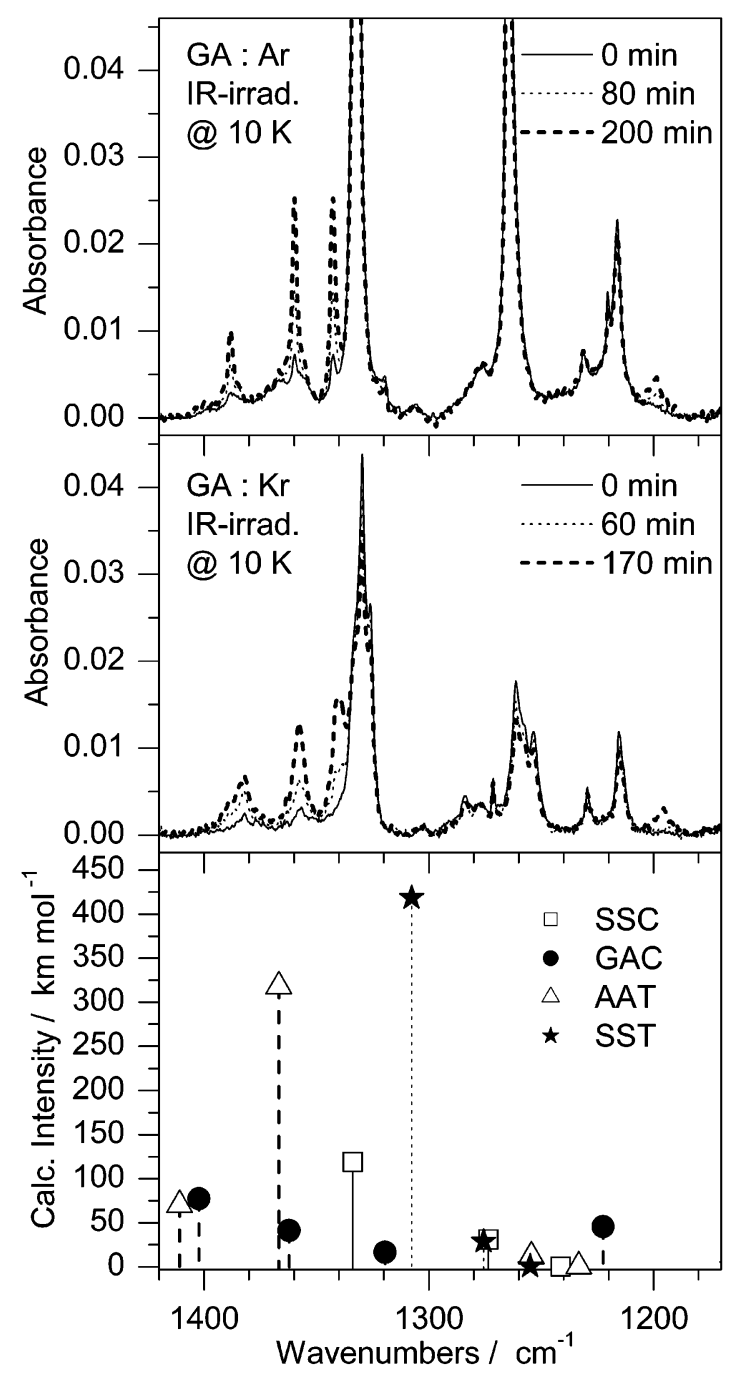

Figure 10. Spectra of GA isolated in argon (upper frame) and krypton (middle frame) matrices and the MP2/aug-cc-pVDZ-predicted (lower frame) spectra for SSC, GAC, AAT, and SST forms (in the vicinity of the strongest predicted band due to the SST form). Calculated frequencies are scaled by 0.993 .

minima (i.e., to catch as many minor forms resulting from the photochemical processes as possible).

The results presented in Figure 9 clearly show that two minor forms of GA were generated upon IR irradiation of the matrices. These two forms were previously populated thermally and identified as GAC and AAT conformers. ${ }^{1}$ The present results are then in consonance with our expectations that, along with the AAT form, the GAC conformer should be produced upon broadband IR irradiation of matrix-isolated GA. On the other hand, there are no indications of any new absorption at the frequency where the SST absorption is expected to appear (between the spectral positions of the bands due to the GAC and AAT forms; see Figure 9).

To come to a definitive conclusion regarding the presence (or absence) in the irradiated matrices of the SST form (and to further confirm the production of both GAC and AAT forms), other spectral regions were analyzed, in particular, those where other strong absorptions of the SST form are predicted. The strongest band in the spectrum of the SST form is expected at $1308 \mathrm{~cm}^{-1}$ (calculated intensity of $418 \mathrm{~km} \mathrm{~mol}^{-1}$ ). This predicted band is distant from any strong vibration in the spectra of the SSC, GAC, and AAT forms (Figure 10). Remarkably, even after prolonged IR irradiation of the matrices, no band

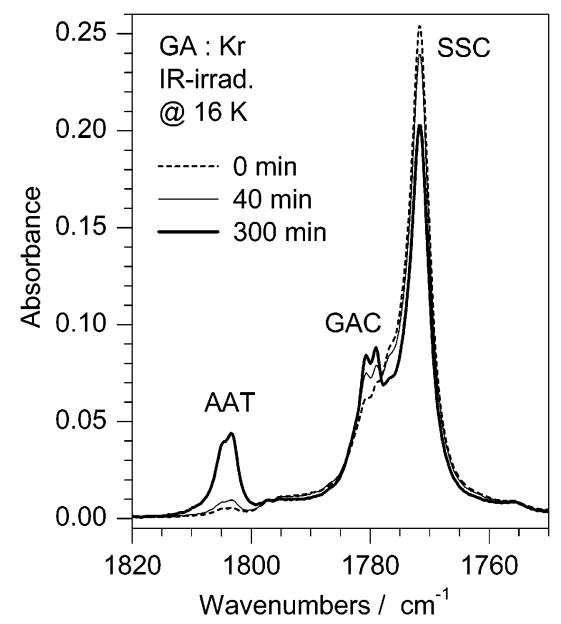

Figure 11. Region of the carbonyl stretching vibration in the IR spectra of matrix-isolated glycolic acid. Dashed line (initial spectrum) corresponds to the sample deposited in $\mathrm{Kr}$ matrix at $16 \mathrm{~K}$. Spectra after shorter (40 min) and longer (300 min) irradiations are presented with thin and thick solid lines, respectively. The absorptions due to different conformers are marked for clarity.

appeared in this frequency region of the experimental spectrum (see Figure 10).

The nonobservation of the SST form indicates that this conformer relaxes to another form after its photoproduction. Taking into consideration the theoretical results discussed in the previous section, we predict that SST shall relax to the AAT form. Note that AAT is the conformer of GA with the highest predicted dipole moment $\left(5.47 \mathrm{D},{ }^{1} 5.45 \mathrm{D}^{19}\right)$. Thus, the AAT form shall be considerably more stabilized in the matrices (compared to the gas phase) than the other conformers, and the energy barrier from SST to AAT shall be effectively lower in matrices than that predicted for the gaseous phase, facilitating the relaxation of the photogenerated SST conformer into AAT.

In conclusion, the experimental data prove that IR irradiation of the matrix-isolated SSC conformer of GA leads to an increase in the populations of both GAC and AAT conformers. The lack of other conformers can be rationalized in terms of their relaxation to one of the three experimentally observed forms (AAC to GAC; ASC to SSC; AST to SST; and SST to either SSC or AAT), as inferred by the systematic theoretical analysis of the possible isomerization pathways.

Once the conformers contributing to the spectra of the irradiated samples are unequivocally identified, a more detailed analysis of the experimental data can be safely undertaken in order to shed some light on the pathways of the photochemical reactions. The first relevant piece of information is the fact that the two observed photoproducts (GAC and AAT) are generated with different relative rates at different stages of the irradiation. This can be clearly proved, for instance, by analysis of the carbonyl stretching spectral region. At the initial stage of the irradiation (see Figure 11), the band due to the GAC form grows at a higher rate than the band ascribed to AAT. After prolonged irradiation, the speed of growth of the GAC-related band decreases comparatively to the growth of the absorption due to the AAT form. However, the absorption band of the GAC form appears on the shoulder of the band due to the main SSC form, which is consumed during IR irradiation. This means lowering of the baseline level for the GAC form with the progress of the irradiation, which in turn may result in an apparent deceleration of the development of the GAC band. To account for this effect, the contribution of the band due to the main SSC form was excluded from the spectra of irradiated samples. The spectrum 


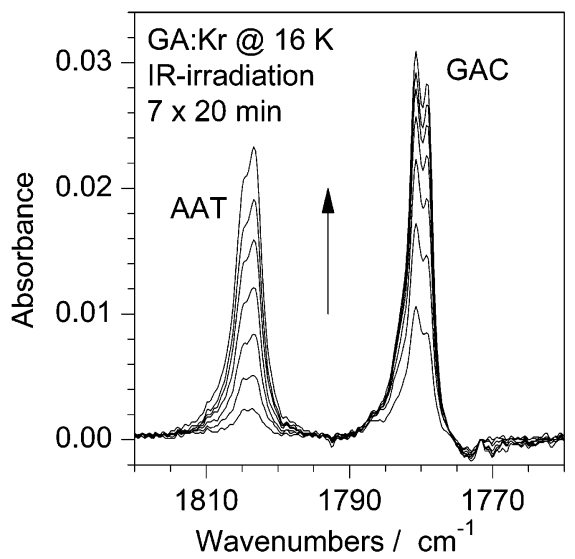

Figure 12. Changes induced in the spectra of the two minor forms of matrix-isolated glycolic acid by IR irradiation. The contribution due to the main (SSC) conformer is normalized by the $\mathrm{C}=\mathrm{O}$ stretching band (peak at $1771.7 \mathrm{~cm}^{-1}$ ) and subtracted from the spectra of irradiated samples. The sample (glycolic acid isolated in krypton matrix at $16 \mathrm{~K}$ ) was consecutively irradiated 7 times, for 20 min each time. The general direction of changes is indicated by the arrow (both bands are growing).

obtained prior to irradiation was scaled down to have the same peak intensity of the carbonyl stretching band due to the SSC form as the spectrum of the irradiated sample (the scaling factor was always different and depended on the irradiation time). Then, this scaled spectrum was subtracted from the spectrum of irradiated sample. The spectra obtained in such a manner (shown in Figure 12) represent the increase in intensity due to the two minor forms after different irradiation times. From such a graph, it is obvious that the population of the AAT conformer increases in a nearly uniform way at all stages of irradiation, while the GAC form is generated more rapidly at the initial stages of irradiation and, as IR irradiation of the matrix proceeds, the growth of the band due to this form slows down.

The IR irradiation of the samples, combined with the abovedescribed procedure of postprocessing of the spectra, turned out to be a powerful analysis tool. It proved not only to provide a clear way for identification of bands due to the photoproduced conformers, but also to enable the extraction of information about experimental frequencies of the bands due to the minor forms that coincide with strong absorptions of the main SSC form and remain undistinguishable in the unprocessed spectra. Particularly spectacular is the result obtained for the $\mathrm{OH}$ stretching region. The spectra recorded before and after IR irradiation were normalized to the same intensity of the SSC band in the fingerprint region and subtracted. The results are presented in Figure 13. In comparison to the $\mathrm{C}=\mathrm{O}$ stretching region, absorptions in the $\mathrm{OH}$ stretching region are much weaker, and the signal-to-noise ratio is lower. Because of these reasons, and for simplicity, only two characteristic irradiation stages are presented in Figure 13. The two $\mathrm{OH}$ stretching vibrations due to the SSC form have theoretically predicted frequencies of 3563.2 and $3570.7 \mathrm{~cm}^{-1}$. In the experimental spectra, these two vibrations appear as a single structured band, which covers the spectral region between 3570 and $3540 \mathrm{~cm}^{-1}$ (see ref 1). Subtraction of this spectral feature allowed the location of the exact position of the absorption due to the $\mathrm{O}_{\mathrm{C}} \mathrm{H}$ stretching band of the GAC form expected in the same region. This band is centered at ca. $3552 \mathrm{~cm}^{-1}$ (see Figure 13), and its position is in good agreement with the theoretical prediction $\left(3569.3 \mathrm{~cm}^{-1}\right)$.

The dependence of the integral intensities of the bands due to the minor forms (Figure 14) on the time of irradiation allows deeper understanding of the mechanism of the photoreaction.

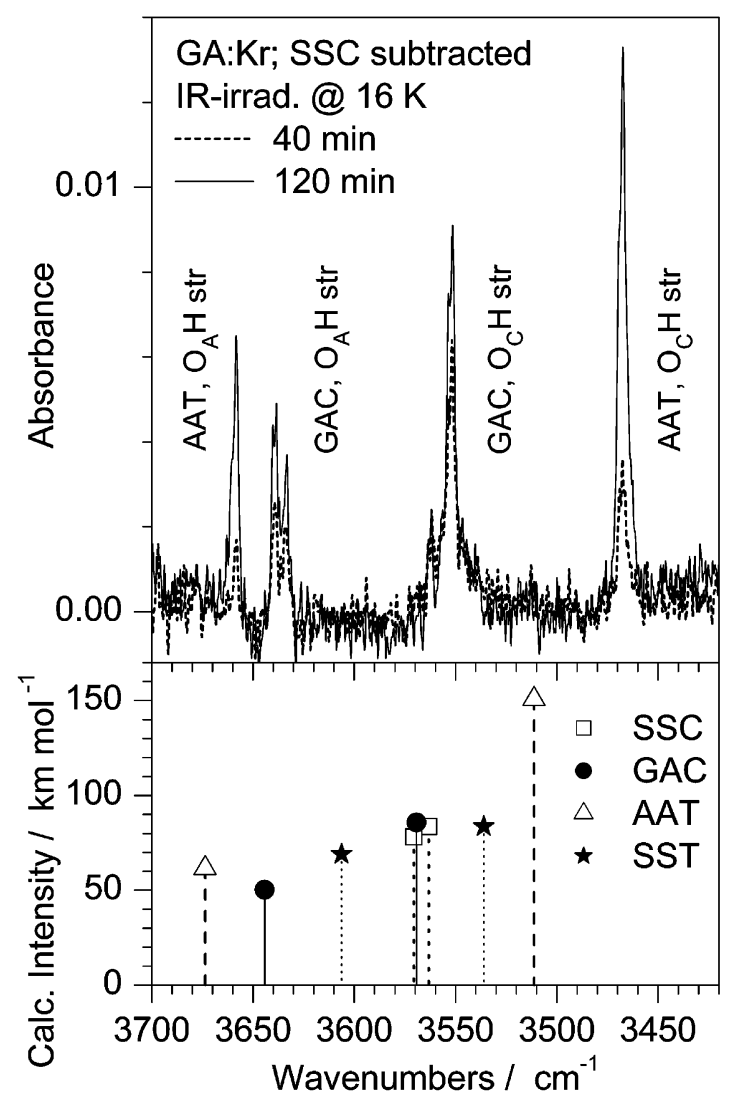

Figure 13. Upper frame: the spectra of glycolic acid isolated in $\mathrm{Kr}$ matrix at $16 \mathrm{~K}$. OH stretching region. The contribution of the SSC form (normalized in the fingerprint region of the spectrum) was eliminated as described in the text. The spectra represent increases in absorptions due to the two minor forms: dashed line, after $40 \mathrm{~min}$; solid line, after 120 min of IR irradiation. $\mathrm{O}_{\mathrm{A}}$ and $\mathrm{O}_{\mathrm{C}}$ designate oxygen atoms from alcohol and carboxylic groups. Lower frame: the spectra theoretically predicted at the MP2/aug-cc-pVDZ level. Calculated frequencies are scaled by 0.955 . Note the absence of bands due to the SST form (nondetectable again) and successful localization of the band due to the $\mathrm{O}_{\mathrm{C}} \mathrm{H}$ stretching vibration (of the GAC form) in the experimental spectrum.

The plots presented in Figure 14 show that the population of conformer GAC grows rapidly after the start of the IR irradiation and shall then correspond to the observed primary photoproduct. The observed nonlinear kinetics for this conformer demonstrates that its population rapidly moves toward a plateau. The GAC form can (i) convert back to the initial SSC form and (ii) react further to conformer AAT. Within the time scale of our experiments, the amount of the AAT form increases linearly with time (the linear fit gives a correlation coefficient equal to 99\% or more for all three probe bands). Extrapolation of the linear fits to the zero-ordinate level shows that the increase of the population of the AAT form starts to be detectable after approximately $10 \mathrm{~min}$ of IR irradiation. On the contrary, extrapolation of the GAC curves confirms that this form starts to grow immediately. All these results indicate that the AAT form appears as a secondary (and ultimate) photoproduct. Together with the analysis of the theoretically calculated potential energy profiles, the experimental data suggest that the primary step of the photoreaction involves rotation of the $\mathrm{O}=$ $\mathrm{C}-\mathrm{C}-\mathrm{O}_{\mathrm{A}}$ dihedral angle. $\mathrm{By}$ this process, the $\mathrm{HO}_{\mathrm{A}} \mathrm{CC}$ dihedral angle adjusts itself automatically from the syn to the gauche position (see the upper frame of Figure 2), and the GAC form is produced. During the second step of the photoreaction, the driving force is the change of the $\mathrm{O}=\mathrm{C}-\mathrm{O}_{\mathrm{C}}-\mathrm{H}$ dihedral angle 


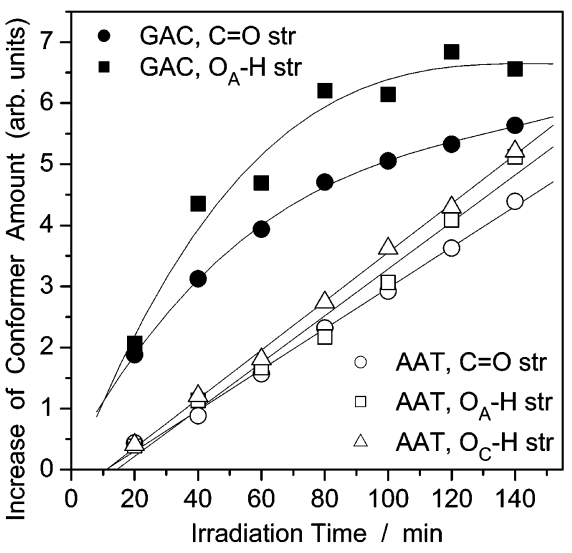

Figure 14. Increase, with time of irradiation, of the amount of the minor conformers (GAC and AAT) with respect to the spectrum before IR irradiation (GA isolated and irradiated in krypton matrix at $16 \mathrm{~K}$ ). The contribution of the SSC form was normalized and subtracted from the spectra of the irradiated samples as described in the text. The amount of each conformer was obtained as the ratio [increase of experimental integral intensity (arb units)]/[calculated intensity of the corresponding vibration]. Theoretical intensities were taken from the MP2/aug-ccpVDZ calculations. $\mathrm{O}_{\mathrm{A}}$ and $\mathrm{O}_{\mathrm{C}}$ designate the oxygen atoms from alcohol and carboxylic groups.

from the cis to the trans position. The alcohol group is again forced to switch its orientation from gauche to anti (see the upper frame of Figure 3 and the animated GIF file provided as Supporting Information Figure 2S), and the AAT form is generated. Although, as it follows from the theoretical discussion in the previous section, photoproduction of all the remaining conformers of GA (either from SSC or GAC) cannot be excluded; no signatures of their generation were discernible in the experimental spectra over the time scale used in our experiments.

\section{Conclusion}

The most stable conformer SSC (characterized by all three torsional angles $\mathrm{HO}_{\mathrm{A}}-\mathrm{C}-\mathrm{C}, \mathrm{O}=\mathrm{C}-\mathrm{C}-\mathrm{O}_{\mathrm{A}}$, and $\mathrm{HO}_{\mathrm{C}}-\mathrm{C}=\mathrm{O}$ equal to $0^{\circ}$ ) was practically the only species trapped in lowtemperature $\mathrm{Ar}$ or $\mathrm{Kr}$ matrices from the room-temperature vapor of glycolic acid. Broad band IR irradiation of the matrix-isolated compound led to the generation of two other conformers, AAT and GAC. Experimental observation of the evolution of a population of the photoproduced conformers as a function of IR-irradiation time, as well as theoretical investigation of possible pathways of conversions from one conformer into the other, allowed identification of the GAC and AAT forms as primary and secondary photoproducts, respectively. Extensive theoretical investigation of the potential energy hypersurface of the compound contributed to better understanding of the interconversion processes between the conformers of glycolic acid.

Acknowledgment. The authors acknowledge Fundação para a Ciência e a Tecnologia (FCT), Lisbon (research project POCTI/QUI/43366/2001 and Grants SFRH/BD/6696/2001 and SFRH/BPD/1661/2000), and FEDER, for financial support.

Dedicated to Prof. Dr. Yuri P. Blagoi on the occasion of his 75th birthday. I.D.R. thanks him for his scientific mentorship over many years of joint work at the Institute for Low Temperature Physics and Engineering, of the National Academy of Sciences of Ukraine.
Supporting Information Available: Figure 1S, comparison of a survey experimental spectrum of glycolic acid isolated in argon matrix with the theoretical spectrum of the SSC conformer calculated at the MP2/aug-cc-pVDZ level; Figure 2S (animated GIF figure), animation of the relaxed potential energy scan for rotation around the $\mathrm{C}-\mathrm{O}_{\mathrm{C}}$ bond in glycolic acid, starting from the second lowest energy form (GAC), demonstrating the triggering of internal rotation in the alcohol $\mathrm{COH}$ group by $\mathrm{COH}$ internal rotation in the carboxyl group; Table $1 \mathrm{~S}$, experimental (argon matrix, $9 \mathrm{~K}$ ) and theoretically calculated (MP2/aug-ccpVDZ) frequencies and intensities of the bands in the IR spectrum of the SSC conformer of glycolic acid and their assignments; Table 2S, internal coordinates used in the normalmode analysis of glycolic acid; Scheme 1S, atom numbering used in definition of internal coordinates. This material is available free of charge via the Internet at http://pubs.acs.org.

\section{References and Notes}

(1) Reva, I. D.; Jarmelo, S.; Lapinski, L.; Fausto, R. Chem. Phys. Lett. 2004, 389, 68-74.

(2) Hollenstein, H.; Schär, R. W.; Schwizgebel, N.; Grassi, G.; Günthard, H. H. Spectrochim. Acta, Part A 1983, 39A, 193-213.

(3) Hollenstein, H.; Ha, T.-K.; Günthard, H. H. J. Mol. Struct. 1986 $146,289-307$.

(4) Godfrey, P. D.; Rodgers, F. M.; Brown, R. D. J. Am. Chem. Soc. 1997, 119, 2232-2239.

(5) Maçôas, E. M. S.; Khriachtchev, L.; Pettersson, M.; Juselius, J.; Fausto, R.; Räsänen, M. J. Chem. Phys. 2003, 119, 11765-11772.

(6) Maçôas, E. M. S.; Khriachtchev, L.; Pettersson, M.; Fausto, R.; Räsänen, M. J. Am. Chem. Soc. 2003, 125, 16188-16189.

(7) Maçôas, E. M. S.; Fausto, R.; Pettersson, M.; Khriachtchev, L.; Räsänen, M. J. Phys. Chem. A 2000, 104, 6956-6961.

(8) Maçôas, E. M. S.; Fausto, R.; Lundell, J.; Pettersson, M.; Khriachtchev, L.; Räsänen, M. J. Phys. Chem. A 2000, 104, 11725-11732.

(9) Maçôas, E. M. S.; Fausto, R.; Lundell, J.; Pettersson, M.; Khriachtchev, L.; Räsänen, M. J. Phys. Chem. A 2001, 105, 3922-3933.

(10) Reva, I. D.; Stepanian, S. G.; Adamowicz, L.; Fausto, R. J. Phys. Chem. A 2001, 105, 4773-4780.

(11) Frisch, M. J.; Trucks, G. W.; Schlegel, H. B.; Scuseria, G. E.; Robb, M. A.; Cheeseman, J. R.; Zakrzewski, V. G.; Montgomery, J. A., Jr.; Stratmann, R. E.; Burant, J. C.; Dapprich, S.; Millam, J. M.; Daniels, A. D.; Kudin, K. N.; Strain, M. C.; Farkas, O.; Tomasi, J.; Barone, V.; Cossi, M.; Cammi, R.; Mennucci, B.; Pomelli, C.; Adamo, C.; Clifford, S.; Ochterski, J.; Petersson, G. A.; Ayala, P. Y.; Cui, Q.; Morokuma, K.; Malick, D. K.; Rabuck, A. D.; Raghavachari, K.; Foresman, J. B.; Cioslowski, J.; Ortiz, J. V.; Stefanov, B. B.; Liu, G.; Liashenko, A.; Piskorz, P.; Komaromi, I.; Gomperts, R.; Martin, R. L.; Fox, D. J.; Keith, T.; Al-Laham, M. A.; Peng, C. Y.; Nanayakkara, A.; Gonzalez, C.; Challacombe, M.; Gill, P. M. W.; Johnson, B. G.; Chen, W.; Wong, M. W.; Andres, J. L.; Head-Gordon, M.; Replogle, E. S.; Pople, J. A. Gaussian 98; Gaussian, Inc.: Pittsburgh, PA, 1998.

(12) Frisch, M. J.; Pople, J. A.; Binkley, J. S. J. Chem. Phys. 1984, 80, 3265-3269.

(13) Kendall, R. A.; Dunning, T. H., Jr.; Harrison, R. J. J. Chem. Phys. 1992, 96, 6796-6806.

(14) Senent, M. L. Mol. Phys. 2001, 99, 1311-1321.

(15) The part of the barrier from $210^{\circ}$ to $285^{\circ}$ has been reoptimized using the very tight convergence criteria of the Gaussian program. The irregularity found in the energy profile is confirmed to be true. It is due to the fact that when the $\mathrm{O}=\mathrm{CCO}_{\mathrm{A}}$ dihedral angle is $270^{\circ}$, the carbonyl group passes through the eclipsed position with one of the hydrogen atoms of the methylene group, and the $\mathrm{C}=\mathrm{O}$ group starts to be accessible for a longrange hydrogen-like interaction with the alcoholic hydrogen. This interaction is energetically favorable, and the alcoholic group leaps towards the carbonyl group (with gain in energy of ca. $10 \mathrm{~kJ} \mathrm{~mol}^{-1}$ ).

(16) Reva, I. D.; Stepanian, S. G.; Adamowicz, L.; Fausto, R. Chem. Phys. Lett. 2003, 374, 631-638.

(17) Ruoff, R. S.; Klots, T. D.; Emilsson, T.; Gutowsky, H. S. J. Chem. Phys. 1990, 93, 3142-3150.

(18) Godfrey, P. D.; Brown, R. D.; Rodgers, F. M. J. Mol. Struct. 1996, $376,65-81$.

(19) Tsipis, A. C.; Tsipis, C. A.; Valla, V. THEOCHEM 2003, 630, 81-100. 